\title{
Chapter 1 \\ Introduction
}

\begin{abstract}
Ensembles on configuration space have wide applicability. They may be used to describe classical, quantum and hybrid classical-quantum systems, physical systems that are deterministic or subject to uncertainty, discrete systems, particles and fields. They also lead to novel reconstructions of quantum theory from physical and geometric axioms. We introduce the basic elements of the theory, discuss a number of classical and quantum examples, and provide an overview of the many generalizations and applications that form the subjects of later chapters. The approach introduces very few physical and mathematical assumptions. The basic building blocks are the configuration space of the physical system, an ensemble of configurations, and dynamics generated from an action principle. An important role is played by the ensemble Hamiltonian which determines the equations of motion. These must satisfy certain requirements which we discuss in detail. We provide examples of classical and quantum systems and show that the primary difference between quantum and classical evolution lies in the choice of the ensemble Hamiltonian.
\end{abstract}

\subsection{The Description of Physical Systems in Terms of Ensembles on Configuration Space}

As noted in the Preface, there is great value in building a formalism for physical theories which is both widely applicable and has a minimal amount of uninterpreted mathematical structure. For example, we may treat the motion of a classical particle subject to an external force using Newtonian, Lagrangian, Hamiltonian or HamiltonJacobi formulations [I, [2]. These different formalisms are equivalent in the sense that they lead to the same predictions, but the amount of effort that goes into solving a particular problem will depend on how well suited the formalism is to that problem. More importantly, different formalisms emphasize different aspects of a system, thus leading to different physical pictures, and will suggest different types of generalizations. For example, the Hamiltonian formalism for classical particles 
was well suited for the early development of quantum mechanics, while Feynmann diagrams in quantum field theory arose from a Lagrangian formulation. Thus, there are very good reasons for considering more than one formulation of a physical theory.

This book develops and applies a formalism for physical theories that is extremely broad in scope - including both classical and quantum mechanics as particular examples - and which is underpinned by a very simple physical picture: ensembles evolving on a configuration space. The mathematical structure is correspondingly simple (simpler than that of $C^{*}$-algebras for example). Yet it is sufficiently nontrivial to be able to guarantee (unlike generalised probabilistic theories for example) the existence of useful objects such as a Lie bracket for observables, canonical transformations, measurement interactions, weak values and thermal ensembles .

The aims of this introductory chapter are to (i) introduce basic elements of the approach, largely via a number of classical and quantum examples, and (ii) preview the many generalisations and applications that form the subjects of later chapters.

\subsection{Basic Concepts and Examples}

The core of the formalism may be summarised in the following

Central idea: Physical systems are described by ensembles on configuration space, the dynamics of which is governed by an action principle.

This central idea makes reference to three familiar concepts: configuration space, ensembles, and Hamilton's principle of least action, briefly discussed in turn below.

The first basic concept is the configuration space of a physical system. For example, in classical mechanics a particle may be in different locations of space, and the configuration space is three-dimensional Euclidean space. By contrast, the configuration space of a two-level system, corresponding to the outcomes of a classical coin toss or measurement on a qubit, is a discrete space with only two elements, such as $\{$ heads, tails $\}$ or $\{$ up, down $\}$. The primary role of a physical theory is to make predictions about system configurations and their evolution. Thus, the mathematical description of a physical system will always involve a choice of configuration space.

The second concept is that of an ensemble on configuration space. The underlying physical idea here is that the formalism should be general enough to cover systems that are subject to uncertainty. Thus, in general, the system will be described by some probability distribution $P$ defined over the configuration space.

The third concept is that the dynamics of the ensemble is specified by an action principle. Since the principle of least action is a generic feature of all known fundamental physical theories, this is not contentious or surprising in itself. However, in the context of ensembles evolving on configuration space, the requirement that the 
probability distribution $P$ remains positive and normalised under evolution will be seen to be an important and nontrivial constraint on allowed ensemble Hamitonians.

As a first example, consider a non-relativistic classical particle of mass $m$ moving in a potential $V(x)$. Our starting point in applying the above concepts will be one of the standard formulations of classical dynamics, the Hamilton-Jacobi equation

$$
\frac{\partial S}{\partial t}+\frac{|\nabla S|^{2}}{2 m}+V=0
$$

A solution $S$ of this equation provides a complete description of the motion when there is no uncertainty: if the particle is known to be at position $x$ at time $t$, then it has momentum $m \dot{x}=\nabla S(x, t)$ and energy $-\partial_{t} S$ [I, 2].

It is not always appreciated that the Hamilton-Jacobi formalism is fundamentally a theory of ensembles [3]. The gradient of $S / m$, in defining a velocity vector at every point on the configuration space, allows any uncertainty regarding the position of the particle to be naturally described by a probability density over the configuration space, $P(x, t)$, with the conservation of probability ensured by a continuity equation of the usual form

$$
\frac{\partial P}{\partial t}+\nabla \cdot\left(P \frac{\nabla S}{m}\right)=0
$$

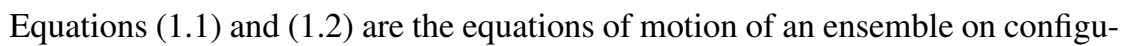
ration space, for the case of a classical particle. But what is the corresponding action principle for the ensemble described by $P$ ? Equivalently, what is the corresponding Langrangian or Hamiltonian that generates these equations of motion? Since $P$ (not $x$ ) is the fundamental quantity describing the ensemble, any suitable 'ensemble Hamiltonian' specifying its evolution should depend on $P$ and some canonically conjugate quantity $\Pi$ [I]. In fact, for the above classical equations of motion one may take $S$ as the quantity canonically conjugate to $P$, and the corresponding classical ensemble Hamiltonian is given by the functional

$$
\mathscr{H}_{C}[P, S]:=\int \mathrm{d} x P\left(\frac{|\nabla S|^{2}}{2 m}+V\right) .
$$

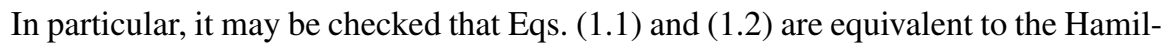
tonian equations of motion

$$
\frac{\partial P}{\partial t}=\frac{\delta \mathscr{H}_{C}}{\delta S}, \quad \frac{\partial S}{\partial t}=-\frac{\delta \mathscr{H}_{C}}{\delta P},
$$

where $\delta / \delta f$ denotes the variational derivative with respect to the function $f^{\boxplus}$ [四]. The classical ensemble Hamiltonian in Eq. ([L.3) may immediately be recognised as the average energy of the ensemble.

\footnotetext{
${ }^{1}$ For readers unfamiliar with variational derivatives, more details are given in App. 因 of this book. It is sufficient to recall here that for $F=\int d x g(x, f, \nabla f)$, one has $\delta F / \delta f=\partial g / \partial f-\nabla \cdot \partial g / \partial(\nabla f)$. The case of discrete configuration spaces is mathematically simpler, as discussed in Sect. 1.3 below.
} 
The formulation of the equations of motion for a classical particle in Eqs. (L.3) and (IL4) may appear unfamiliar, and it certainly does not appear in standard textbooks on mechanics. However, the form of the classical ensemble Hamiltonian $\mathscr{H}_{C}$ is in fact well known in the theory of ideal fluids (dating back to the 19 century), where the quantity $S / m$ is reinterpreted as a velocity potential [4]. Moreover, the fundamental nature of this formulation quickly becomes apparent when it is realised that the equations of motion for a quantum particle have a remarkably similar form.

In particular, let $\psi(x)$ denote the wave function for a quantum particle of mass $m$ subject to potential $V(x)$, and define the real functions $P$ and $S$ on configuration space via the polar decomposition $\psi=\sqrt{P} \mathrm{e}^{-\mathrm{i} S / \hbar}$. The equations of motion for $P$ and $S$ then follow via substitution into the standard Schrödinger equation for $\psi$, yielding the continuity equation

$$
\frac{\partial P}{\partial t}+\nabla \cdot\left(P \frac{\nabla S}{m}\right)=0
$$

as before, and the modified Hamilton-Jacobi equation

$$
\frac{\partial S}{\partial t}+\frac{|\nabla S|^{2}}{2 m}+V-\frac{\hbar^{2}}{2 m} \frac{\nabla^{2} P^{1 / 2}}{P^{1 / 2}}=0
$$

These equations are equivalent to the Hamiltonian equations of motion

$$
\frac{\partial P}{\partial t}=\frac{\delta \mathscr{H}_{Q}}{\delta S}, \quad \frac{\partial S}{\partial t}=-\frac{\delta \mathscr{H}_{Q}}{\delta P},
$$

analogous to Eqs. (ㅍ.4) above, where $\mathscr{H}_{Q}$ is the quantum ensemble Hamiltonian

$$
\mathscr{H}_{Q}[P, S]:=\int \mathrm{d} x P\left(\frac{|\nabla S|^{2}}{2 m}+V+\frac{\hbar^{2}}{8 m} \frac{|\nabla P|^{2}}{P^{2}}\right) .
$$

Eqs. (ㅍ.5) and (ㄷ.6), together with the ensemble Hamiltonian $\mathscr{H}_{Q}$, were first given by Madelung in 1926 [5]. Madelung further showed that

$$
\mathscr{H}_{Q}[P, S]=\int \mathrm{d} x \psi^{*}(x)\left(-\frac{\hbar^{2}}{2 m} \nabla^{2}+V\right) \psi(x)=\left\langle-\frac{\partial S}{\partial t}\right\rangle,
$$

which may be recognised as the average quantum energy for the wave function $\psi$. Thus, just as for the classical case, the ensemble Hamiltonian is equal to the average energy of the ensemble, and also to the average of $-\partial_{t} S$. Further, in the limit $\hbar \rightarrow 0$ one has $\mathscr{H}_{Q} \rightarrow \mathscr{H}_{C}$, and hence the quantum and classical equations of motion become identical.

The above examples show that the dynamics of classical and quantum nonrelativistic particles can be treated using the common framework of ensembles on configuration space, where the primary difference between quantum and classical evolution lies in the choice of the ensemble Hamiltonian. We explore this framework a little further in this chapter, to give examples for discrete configuration spaces and 
to note some fundamental properties of ensemble Hamiltonians. In later chapters we will considerably develop the framework, to give a unified treatment of concepts such as observables, fields, interaction, measurement, superselection, weak values, and thermodynamics on configuration space, without reference to any particular theory. This general framework is capable of attacking a number of interesting problems that either cannot be formulated in the language used by other theories or are extremely difficult to formulate, such as consistent descriptions of measurements on a quantum system by a classical measuring device and the coupling of quantum fields to a classical gravitational field. It is also shown to be a powerful starting point for the derivation of the quantum formalism from suitable axioms (see Sect. $\mathbb{L} .5$ for an indication of the material covered in later chapters).

\subsection{Further Examples: Discrete Configuration Spaces}

The formalism of ensembles on configuration space is very broadly applicable. It is worthwhile in this introductory chapter to see how it is able to incorporate classical and quantum systems with discrete configuration spaces. Indeed, the formalism is somewhat simpler in this case, than for particles in the previous section, as no variational derivatives are involved.

\subsubsection{Classical Rate Equations}

Consider the evolution of a classical probability distribution $P \equiv\left\{P_{j}\right\}$ on a discrete set of points labelled by $1,2,3, \ldots$. This is a ubiquitous problem in the theory of stochastic processes [6]. If $T_{j k}$ denotes the rate at which probability flows from site $k$ to site $j$, then the evolution of $P$ is given by the transition rate equation

$$
\frac{\partial P_{j}}{\partial t}=\sum_{k}\left(T_{j k} P_{k}-T_{k j} P_{j}\right)
$$

(sometimes called a classical master equation or Kolmogorov equation). The first part of the sum generates the total probability flowing into site $j$, and the second part generates the total probability flowing out. Note that the transition rates can depend on $P$, and that $T_{k k}$ may be chosen arbitrarily as it makes no contribution to the rate equation. The equation is often represented pictorially by a transition graph: the points correspond to the vertices of the graph, and for each pair of vertices $(j, k)$ there is a directed edge from vertex $k$ to vertex $j$ labeled by $T_{j k}$ [6].

Equation (디) is a discrete analog of the continuity equation (ㅍ.2) for probability flow on a continuous configuration space, and similarly ensures the conservation of probability. This may be checked via summation of both sides over $j$, and swapping the dummy indices in the second term. 
To model this case within the formalism of ensembles on configuration space, we require the existence of a canonically conjugate quantity $S \equiv\left\{S_{j}\right\}$ on the configuration space, and a suitable ensemble Hamiltonian $H(P, S)$, such that the equations of motion are given by

$$
\frac{\partial P_{j}}{\partial t}=\frac{\partial H}{\partial S_{j}}, \quad \frac{\partial S_{j}}{\partial t}=-\frac{\partial H}{\partial P_{j}} .
$$

It is straightforward to construct such a Hamiltonian system: choose

$$
H(P, S)=\sum_{j, k} T_{j k} P_{k}\left(S_{j}-S_{k}\right) .
$$

The Hamiltonian equations of motion then generate the transition rate equation (II.JI) as desired. It should be noted that this is a purely formal construction: transition rate equations are typically obtained from an underlying microscopic description that incorporates the fundamental physics, with a given transition rate equation being compatible with many possible such descriptions. Nevertheless, the construction shows that such equations, while only providing a phenomenological model, can easily be incorporated into the formalism of ensembles on configuration space.

An important feature of the above ensemble Hamiltonian $H(P, S)$ is that it only depends on $S$ via the differences between its components. It will be seen in Sect. 1.4 that this feature is generic for discrete configuration spaces, and is related to the conservation of probability. It may further be noted that, for the special case of constant transition rates, the above ensemble Hamiltonian further generates the equation

$$
\frac{\partial S_{j}}{\partial t}=-\sum_{k} T_{j k}\left(S_{j}-S_{k}\right)
$$

for the conjugate quantity $S$. As this is independent of the evolution of $P$, it has no direct physical import (at least, not without a specific underlying microscopic model). However, by multiplying this equation by $P_{j}$ and summing over $j$ one immediately obtains the connection

$$
H(P, S)=-\sum_{j} P_{j} \frac{\partial S_{j}}{\partial t}=\left\langle-\frac{\partial S}{\partial t}\right\rangle
$$

between the ensemble Hamiltonian and the average of $-\partial_{t} S$, which similarly held for the classical and quantum ensembles of the previous section. Hence, $-\partial_{t} S_{j}$ may be interpreted as the energy associated with site $j$ in this case. It will be seen in Sect. ‥4 that this connection is guaranteed for ensemble Hamiltonians satisfying a homogeneity property. Other physical roles for $S$ will be seen in later chapters (most notably in regard to generating physical transformations of the ensemble such as translations and reflections). 


\subsubsection{Finite-Dimensional Quantum Systems}

For quantum systems evolving on a finite Hilbert space, the configuration space may be chosen to be the set of discrete outcomes of any complete measurement on the system. For example, for nondegenerate Hermitian operator $\hat{A}$, with orthormal eigenstates $\left\{\left|a_{j}\right\rangle\right\}$, the configuration space is $\left\{a_{j}\right\}$. The choice of measurement is in principle arbitrary, and corresponds to the choice of the 'computational basis' in quantum computing theory.

For quantum state $|\psi\rangle=\sum_{j} \psi_{j}\left|a_{j}\right\rangle$, define $P_{j}$ and $S_{j}$ via the polar decomposition $\psi_{j}=\sqrt{P_{j}} \mathrm{e}^{\mathrm{i} S_{j} / \hbar}$. Thus, $P \equiv\left\{P_{j}\right\}$ is the probability distribution on configuration space for the ensemble of systems described by $|\psi\rangle$. Guided by the previous examples, an obvious candidate for the corresponding ensemble Hamiltonian is the average energy of the ensemble,

$$
H(P, S):=\langle\psi|\hat{H}| \psi\rangle=\sum_{j, k} h_{j k} \sqrt{P_{j} P_{k}} \mathrm{e}^{-\mathrm{i}\left(S_{j}-S_{k}\right) / \hbar},
$$

where $\hat{H}$ is the quantum Hamiltonian operator and $h_{j k}:=\left\langle a_{j}|\hat{H}| a_{k}\right\rangle$. It is then straighforward to check that the Hamiltonian equations of motion (피) are indeed equivalent to the real and imaginary parts of the Schrödinger equation

$$
\hat{H}|\psi\rangle=\mathrm{i} \hbar \frac{\partial|\psi\rangle}{\partial t}
$$

It may also be checked that Eq. (IL.14) holds for the average energy, i.e, $H(P, S)=$ $\left\langle-\partial_{t} S\right\rangle$.

Just as for the classical rate equations, the ensemble Hamiltonian in Eq. (I. I5) only depends on differences between the components of $S$, implying that the equations of motion are invariant under the addition of an arbitrary constant to the components of $S$. In quantum mechanics this is usually interpreted in terms of the physical irrelevance of the global phase of the wave function. However, in the more general formalism of ensembles on configuration space, this property follows as a fundamental consequence of the conservation of probability (see Sect. L.4).

It is straightforward to generalise the above results to any complete basis set $\{|a\rangle\}$ for a quantum system, whether finite or infinite, discrete or continuous, orthonormal or otherwise [7] (one only requires that $\sum_{a}|a\rangle\langle a|=\hat{1}$, with summation replaced by integration over any continuous ranges of $a$ ). One may similarly generalise the results to quantum field theory (see for example Chaps. [1 and [1], where fields are discussed). 


\subsection{Fundamental Properties of Ensemble Hamiltonians}

We may abstract from the examples of the previous sections to rewrite the central idea in Sect. ․2 more formally, as

Central idea (formal version): Physical systems are described by a probability density $P$ on configuration space, a canonically conjugate quantity $S$, and an ensemble Hamiltonian $\mathscr{H}(P, S)$.

This idea is very simple but surprisingly powerful. It not only incorporates and unifies the description of standard classical and quantum dynamics, but provides a general and useful framework for formulating more general physical theories, as will be seen in later chapters.

The state of the system is described by the configuration ensemble $(P, S)$. The role of the ensemble Hamiltonian $H(P, S)$ is to specify the dynamics of this ensemble ${ }^{\square}$. Hence, in particular, it must evolve probabilities to probabilities. This places some important physical constraints on the choice of possible ensemble Hamiltonians, as will now be discussed.

\subsubsection{Conservation of Probability}

Probabilities must always sum to unity, and hence the evolution of an ensemble must respect this property. It will be shown here that the conservation of probability corresponds to invariance of the dynamics under the addition of an arbitrary constant to $S$.

First, for an ensemble on a discrete configuration space with probability distribution $P_{j}$, note that conservation of probability is equivalent to $\sum_{j} P_{j}(t)=\sum_{j} P_{j}(t+$ $\varepsilon)=1$. Hence, to first order in $\varepsilon$, one has via Eq. (ㅁ. $\mathbb{l}$ ) that

$$
0=\sum_{j}\left[P_{j}(t+\varepsilon)-P_{j}(t)\right]=\sum_{j} \varepsilon \frac{\partial P_{j}}{\partial t}=\varepsilon \sum_{j} \frac{\partial \mathscr{H}}{\partial S_{j}}=\mathscr{H}(P, S+\varepsilon)-\mathscr{H}(P, S) .
$$

Hence, by considering a sequence of infinitesimal evolutions, it follows that

$$
\mathscr{H}(P, S+c)=\mathscr{H}(P, S)
$$

as claimed. In particular, this implies for discrete configuration spaces that the ensemble Hamiltonian only depends on $S$ via the differences $M_{j k}:=S_{j}-S_{k}$ : only relative values of $S$ are physically relevant. Note for quantum systems that this property

\footnotetext{
${ }^{2}$ For continuous configuration spaces $P$ and $S$ are functions, and hence one should more properly write the ensemble Hamiltonian as a functional, $H[P, S]$, in this case. However, it is convenient to use the notation $H(P, S)$ when referring to the general case.
} 
corresponds to (and explains) the irrelevance of a global phase factor of the quantum state (see previous section).

It follows, writing $\mathscr{H}(P, S) \equiv f(S, M)$ for some function $f$, that the equation of motion for $P$ reduces to the form of a transition rate equation,

$$
\frac{\partial P_{j}}{\partial t}=\sum_{k}\left(\frac{\partial f}{\partial M_{j k}}-\frac{\partial f}{\partial M_{k j}}\right)=\sum_{k}\left(T_{j k} P_{k}-T_{k j} P_{j}\right)
$$

with corresponding transition rates

$$
T_{j k}:=\left(P_{k}\right)^{-1} \frac{\partial f}{\partial M_{j k}}
$$

(and $T_{j k}:=0$ for $P_{k}=0$ ). Note that, unlike the classical rate equation in Eq. (I.TO)), these transition rates will generally depend on $S$. In particular, for the discrete quantum ensemble Hamiltonian in Eq. (I.T5) one finds

$$
T_{j k}=\hbar^{-1} \sqrt{P_{j} / P_{k}} \operatorname{Im}\left\{h_{j k} \mathrm{e}^{-\mathrm{i}\left(S_{j}-S_{k}\right) / \hbar}\right\} .
$$

Such quantum transition rate equations have been used by Bell to formulate a theory of beables for fermionic fields [8], and by others to formulate modal interpretations of quantum dynamics [9].

For a continuous configuration space the ensemble Hamiltonian is more properly written as a functional, $H[P, S]$. Replacing partial derivatives by variational derivatives and summation by integration in the derivation of Eq. ([.18), one then obtains ${ }^{\text {b] }}$

$0=\int \mathrm{d} x[P(x, t+\varepsilon)-P(x, t)]=\int \mathrm{d} x \varepsilon \frac{\partial P}{\partial t}=\varepsilon \int \mathrm{d} x \frac{\delta \mathscr{H}}{\delta S}=\mathscr{H}[P, S+\varepsilon]-\mathscr{H}[P, S]$,

to first order in $\varepsilon$. Thus, in complete analogy to the discrete case in Eq. ([.]8), we have

$$
\mathscr{H}[P, S+c]=\mathscr{H}[P, S] .
$$

Note that this property is guaranteed for the classical and quantum ensemble Hamiltonians $\mathscr{H}_{C}[P, S]$ and $\mathscr{H}_{Q}[P, S]$, in Eqs. ([L.3) and ([L.8) respectively, because they only depend on $S$ via its derivative $\nabla S$. The general result is worth highlighting:

\section{Conservation of probability implies that}

- The ensemble Hamiltonian is invariant under $S \rightarrow S+c$ for any constant $c$.

- Only relative values of $S$ have dynamical significance.

\footnotetext{
${ }^{3}$ Here the defining property of the variational derivative, $F[f+\delta f]-F[f]=\int d x(\delta F / \delta f) \delta f$ for arbitrary infinitesimal variations $\delta f$, has been used (see App. 因 of this book).
} 


\subsubsection{Positivity of Probability}

A second fundamental property of probability is that it is positive, i.e., $P \geq 0$. This enforces a further nontrivial constraint on the ensemble Hamiltonian. For example, while $H(P, S)=S_{2}-S_{1}$ satisfies Eq. (‥8) , and hence conserves probability, the equations of motion (ILII) yield $P_{1}(t)=P_{1}(0)-t$, which eventually becomes negative.

A necessary condition for ensuring positivity is obtained by expanding the probability about a given time $t$ :

$$
P(t+\varepsilon)=P(t)+\varepsilon \frac{\partial P}{\partial t}+\frac{1}{2} \varepsilon^{2} \frac{\partial^{2} P}{\partial t^{2}}+\ldots
$$

In particular, one must have $\frac{\partial P}{\partial t}=0$ and $\frac{\partial^{2} P}{\partial t^{2}} \geq 0$ whenever $P(t)=0$, as otherwise $P$ will be negative at times either just before or just after time $t$. The first of these conditions corresponds to the constraints

$$
\frac{\partial \mathscr{H}}{\partial S_{j}}=0 \text { for } P_{j}=0, \quad \frac{\delta \mathscr{H}}{\delta S}=0 \text { for } P(x)=0,
$$

on the ensemble Hamiltonian, for discrete and continuous configuration spaces respectively. One can similarly write down a corresponding constraint for the second condition, involving derivatives of the ensemble Hamiltonian to second order.

For example, for classical and quantum particles the positivity condition ([L.25) reduces, via the continuity equation (․2), to

$$
\frac{\partial P}{\partial t}=-\nabla \cdot\left(\frac{P \nabla S}{m}\right)=-\frac{P \nabla^{2} S}{m}-\frac{\nabla P \cdot \nabla S}{m}=0 \text { for } P(x)=0 .
$$

To verify this condition holds, note that if $P(x)=0$ for some point $x$ (at some fixed time $t$ ), then this is necessarily a global minimum of $P(x)$, implying that $\nabla P=0$ at point $x$. Thus $\frac{\partial P}{\partial t}=0$ as required. One may simlarly check that $\frac{\partial^{2} P}{\partial t^{2}} \geq 0$ (see Chap. (2).

For the discrete examples in section 1.3, positivity condition ([L.25) reduces, via either of the transition rate equations (‥T) and (‥T), to $\sum_{k} T_{j k} P_{k}=0$ for $P_{j}=0$. For the classical transition rate equation (1.10) the condition must be checked in each case, but will of course hold for equations derived from physical microscopic models. In the quantum case its validity follows immediately from Eq. (‥21) for the quantum transition rates - indeed, for this case one has the stronger result $T_{j k}=0$ for $P_{j}=0$. 


\subsubsection{Homogeneity}

The examples of ensemble Hamiltonians in Eqs. (ㄸ.3), (ㄸ.8) and (ㅁ.15) all satisfy the simple homogeneity property

$$
\mathscr{H}(\lambda P, S)=\lambda \mathscr{H}(P, S), \quad \lambda \geq 0 .
$$

This property, while not mathematically necessary, is of fundamental interest due to its physical implications.

First, note that taking the derivative of Eq. (L.27) with respect to $\lambda$ and evaluating the result at $\lambda=1$ yields

$$
\mathscr{H}(P, S)=\left\langle-\frac{\partial S}{\partial t}\right\rangle
$$

for both discrete and continuous configuration spaces (using the Hamiltonian equations of motion $\partial_{t} S_{j}=-\partial \mathscr{H} / \partial P_{j}$ and $\partial_{t} S=-\delta \mathscr{H} / \delta P$ respectively). Second, consider the decomposition of $P$ into a mixture of two ensembles on the configuration space, i.e.,

$$
P=w_{1} P^{(1)}+w_{2} P^{(2)}, \quad w_{1}, w_{2} \geq 0, \quad w_{1}+w_{2}=1
$$

It then follows from Eq. ([1.28), assuming a discrete configuration space for definiteness, that

$$
\begin{aligned}
\mathscr{H}(P, S) & =-\sum_{j} P_{j} \frac{\partial S_{j}}{\partial t}=-w_{1} \sum_{j} P_{j}^{(1)} \frac{\partial S_{j}}{\partial t}-w_{2} \sum_{j} P_{j}^{(2)} \frac{\partial S_{j}}{\partial t} \\
& =w_{1} \mathscr{H}\left(P^{(1)}, S\right)+w_{2} \mathscr{H}\left(P^{(2)}, S\right) .
\end{aligned}
$$

Hence, the numerical value of the ensemble Hamiltonian is just the weighted average of the values for the two subensembles, as required for interpreting it as an average energy. It further follows from Eq. (‥28) that $-\partial_{t} S$ may be interpreted as a corresponding local energy density on the configuration space (however, note that the first result does not require that this local energy density is a physical energy see also Sect. (2.4.2). Thus:

\section{The homogeneity property implies that}

- The ensemble Hamiltonian can be interpreted as the average energy of the ensemble.

- The quantity $-P \partial_{t} S$ is a local energy density for the ensemble.

We will see in Chap. \ that the above results leads to a substantial generalization of the concept of weak values in quantum mechanics, to any theory with observables satisfying the homogeneity property. 
The usefulness of similar homogeneity properties (for evolving the quantum wave function) has been noted previously in nonlinear extensions of quantum theory [10, 11, 12]. The above results show that homogeneity can be motivated at the more general level of ensembles on configuration space, without any reference to wave functions or to quantum mechanics. In Chaps. 1$]$ and $\mathbf{Q}$ this assumption will also be seen to be important for the consistent description of independently evolving ensembles.

\subsection{Outline of This Book}

In the following chapters, we will further develop the general formalism of ensembles on configuration space, and apply it in a wide variety of contexts, including measurement, thermodynamics, axiomatic approaches to quantum mechanics, and the coupling of classical spacetime to quantum matter. Along the way many concepts in classical and quantum theory will be unified and generalised via the overarching framework provided by the configuration ensemble approach. While some of the material has previously appeared in some form in the literature, as indicated below, many results are presented for the first time.

We continue Part I of the book, i.e., the exposition of general properties of the formalism, in Chaps. ㅁ-7. First, in Chap. ㅁ we introduce a definition of observables for arbitrary ensembles on configuration space, and show that these encompass both classical and quantum observables [7, 13, 14, 24]. We also show that the formalism allows for the generalisation of certain quantum concepts, such as eigenstates, eigenvalues, weak values and transition probabilities, to arbitrary configuration ensembles.

In Chap. 13, we describe composite systems via joint ensembles [113, [14]. These may consist of subsystems which are, e.g., independent or entangled, interacting or noninteracting. A precise formulation of these properties is provided. We define the extension of single-system observables to joint ensembles, and discuss their algebraic and separability properties. The rest of this chapter is devoted to a description of measurement interactions, starting with basic measurement models, then more elaborate ones describing weak measurements and measurement-induced collapse.

In Chap. 团, we consider mixtures of configuration space ensembles, and generalise the quantum notions of 'proper' and 'improper' mixtures. With the help of such mixtures it becomes possible to unify and generalise traditional classical and quantum approaches to thermodynamics, via the definition of suitable 'thermal mixtures.' Our formulation is very different to standard approaches based on the maximum entropy principle, and is of particular interest in providing a novel Hamilton-Jacobi picture of classical thermodynamics.

In Part II of the book, comprising Chaps. [-Z, we show how the configuration ensemble approach provides a natural starting point for three very different axiomatic approaches to quantum mechanics. First, in Chap. \$ we introduce a quantization procedure for classical ensembles which provides an alternative to standard quanti- 
zation methods [15, 16, 17, 18, 19]. We show that it is possible to formulate an exact form of the uncertainty principle, which provides the single key element that is needed for moving from the equations of motion of a classical ensemble to those of a quantum ensemble. The quantization procedure is used to derive the Schrödinger equation and bosonic field equations.

In Chap. 6, we look at the geometry of ensembles on configuration space for both discrete and continuous systems [20, 21, 22, 23]. We show that the theory has a rich geometry, and that the geometrical structures natural to the space can be used to obtain a geometrical reconstruction of quantum mechanics. The basic structures used are the natural metric on the space of probabilities (information geometry) and the description of dynamics using a Hamiltonian formalism (symplectic geometry); requirements of consistency then lead to a Kähler geometry. This geometrical reconstruction of quantum mechanics has some remarkable features. The wave functions of quantum mechanics appear as the natural complex coordinates of the Kähler space, the full group of unitary transformations is derived based on consistency requirements, and a Hilbert space may be associated with the Kähler space of the theory, leading to the standard version of quantum theory.

In Chap. Z, we consider local representations of rotations on discrete configuration spaces, focusing in particular on ensembles of either one or two spin-half systems, which we call rotational bits or 'robits'. In the case of a single robit, the theory is equivalent to that of a single quantum mechanical qubit. The description of a pair of robits is more complicated, in that requirements of locality and subsystem independence must be taken into account. We show that in this case, in addition to a theory which is equivalent to the quantum theory of a pair of qubits, it may also be possible to have non-quantum local models.

Part III of the book, comprising Chaps. 8 and 9 , deals with hybrid classicalquantum systems [13, 14, 24, 25, 26]. The problem of defining hybrid systems comprising quantum and classical components is highly nontrivial, and the approaches that have been proposed to solve this problem run into various types of fundamental difficulties. The formalism of configuration-space ensembles is able to overcome many of these difficulties, allowing for a general and consistent description of interactions between quantum and classical ensembles. In Chap. \&, we discuss general properties of hybrid ensembles and consider various applications: measurements of a quantum system by a classical apparatus, scattering, harmonic oscillators, and hybrid Wigner functions. In Chap. Q , we focus on consistency requirements for quantum-classical interactions. We show how the configuration ensemble approach is able to satisfy desirable properties such as a Lie algebra of observables and Ehrenfest relations, while evading no-go theorems based in part on such properties. We then discuss locality aspects of the approach and present a measurement model of wide applicability.

Finally, Part IV, comprising Chaps. 10 and W1, is devoted to ensembles of classical gravitational fields and their interaction with quantum fields [18, 19, 27, 28]. After considering the case of pure gravity in Chap. 10, which we illustrate with the example of ensembles of black holes, Chap. $\square$ discusses the coupling of classical gravitational fields to quantum matter fields. In the standard approach to this 
problem (i.e., semiclassical gravity), the energy momentum tensor that serves as the source of the Einstein equations is replaced by the expectation value of the energy momentum operator with respect to a particular quantum state. This approach, however, presents a number of well known difficulties. We show that a viable alternative is provided by the use of ensembles on configuration space, which leads to a theory that is consistent and which does not have any of the problems of semiclassical gravity. We illustrate the power of the approach with two examples: a cosmological model which consists of a closed Robertson-Walker universe with a massive quantum scalar field and a classical CGHS black hole in a collapsing geometry interacting with a quantized scalar field.

\section{References}

1. Goldstein, H.: Classical Mechanics. Addison-Wesley, New York (1950)

2. Synge, J.L.: Classical Dynamics. In: Flügge, S. (ed.) Encyclopedia of Physics, Vol. III/1, pp. 1-225. Springer-Verlag, Berlin (1960)

3. Landauer, R.: Path concepts in Hamilton-Jacobi theory. Am. J. Phys. 20, 363-367 (1952)

4. Zakharov, V.E., Kuznetsov, E. A.: Hamiltonian formalism for nonlinear waves. Physics Uspekhi 40, 1087-1116 (1997)

5. Madelung, E.: Quantentheorie in hydrodynamischer Form. Z. Physik 40, 322-326 (1926)

6. Beichelt, F.: Stochastic Processes in Science, Engineering and Finance, chapter 5. Taylor \& Francis, Boca Raton, Florida (2006)

7. Hall, M.J.W.: Superselection from canonical constraints. J. Phys. A 27, 7799-7811 (2004)

8. Bell, J.S.: Beables for quantum field theory. In: Bell, M., Gottfried, K., Veltman M. (eds.) John S. Bell on the Foundations of Quantum Mechanics, pp. 159-166. World Scientific, Singapore (2001)

9. Gambetta J., Wiseman, H.M.: Modal dynamics for positive operator measures. Found. Phys. 34, 419-448 (2004); see also references therein.

10. Haag R., Bannier, U.: Comments on Mielnik's generalized (non linear) quantum mechanics. Commun. Math. Phys. 60, 1-6 (1978)

11. Kibble, T.W.B.: Relativistic models of nonlinear quantum Mechanics. Commun. Math. Phys. 64, 73-82 (1978)

12. Weinberg, S.: Testing quantum mechanics. Ann. Phys. N.Y. 194, 336-386 (1989)

13. Hall, M.J.W., Reginatto, M.: Interacting classical and quantum ensembles. Phys. Rev. A 72, $062109(2005)$

14. Hall, M.J.W.: Consistent classical and quantum mixed dynamics. Phys. Rev. A 78, 042104 (2008)

15. Hall, M.J.W., Reginatto, M.: Schrödinger Equation from an Exact Uncertainty Principle. J. Phys A 35, 3289-3303 (2002)

16. Hall, M.J.W., Reginatto, M.: Quantum Mechanics from a Heisenberg-type Equality. Fortschr. Phys. 50, 646-651 (2002)

17. Hall, M.J.W., Kumar, K., Reginatto, M.: Bosonic field equations from an exact uncertainty principle. J. Phys A 36, 9779-9794 (2003)

18. Reginatto, M.: Exact Uncertainty Principle and Quantization: Implications for the Gravitational Field. Brazilian Journal of Physics 35, 476-480 (2005)

19. Hall, M.J.W.: Exact uncertainty approach in quantum mechanics and quantum gravity. Gen. Relativ. Gravit. 37, 1505-1515 (2005)

20. Reginatto, M., Hall, M.J.W.: Quantum theory from the geometry of evolving probabilities. In: Goyal, P., Giffin, A., Knuth, K.H., Vrscay, E. (eds.) Bayesian Inference and Maximum Entropy Methods in Science and Engineering, 31st International Workshop on Bayesian Inference and Maximum Entropy Methods in Science and Engineering, Waterloo, Canada, 10-15 
July 2011. AIP Conference Proceedings, vol. 1443, American Institute of Physics, Melville, New York (2012)

21. Reginatto, M., Hall, M.J.W.: Information geometry, dynamics and discrete quantum mechanics. In: von Toussaint, U. (ed.) Bayesian Inference and Maximum Entropy Methods in Science and Engineering, 32nd International Workshop on Bayesian Inference and Maximum Entropy Methods in Science and Engineering, Garching, Germany, 15-20 July 2012. AIP Conference Proceedings, vol. 1553, American Institute of Physics, Melville, New York (2013)

22. Reginatto, M.: From probabilities to wave functions: A derivation of the geometric formulation of quantum theory from information geometry. J. Phys.: Conf. Ser. 538, 012018 (2014)

23. Reginatto, M.: The geometrical structure of quantum theory as a natural generalization of information geometry. In: Mohammad-Djafari. A., Barbaresco, F. (eds) Bayesian Inference and Maximum Entropy Methods in Science and Engineering, 34th International Workshop on Bayesian Inference and Maximum Entropy Methods in Science and Engineering, Clos Lucé, Amboise, France, 21-26 September 2014. AIP Conference Proceedings, vol. 1641, American Institute of Physics, Melville, New York (2015)

24. Reginatto, M., Hall, M.J.W.: Quantum-classical interactions and measurement: a consistent description using statistical ensembles on configuration space. J. Phys.: Conf. Ser. 174, 012038 (2009)

25. Chua, A.J.K., Hall, M.J.W., Savage, C.M.: Interacting classical and quantum particles. Phys. Rev. A 85, 022110 (2011)

26. Hall, M.J.W., Reginatto, M., Savage, C.M.: Nonlocal signaling in the configuration space model of quantum-classical interactions. Phys. Rev. A 86, 054101 (2012)

27. Albers, M., Kiefer, C., Reginatto, M.: Measurement analysis and quantum gravity. Phys. Rev. D 78, 064051 (2008)

28. Reginatto, M.: Cosmology with quantum matter and a classical gravitational field: the approach of configuration-space ensembles. J. Phys.: Conf. Ser. 442, 012009 (2013) 